\title{
Phenylalanine hydroxylase deficiency: diagnosis and management guideline
}

\author{
Jerry Vockley, MD, PhD ${ }^{1,2}$, Hans C. Andersson, $\mathrm{MD}^{3}$, Kevin M. Antshel, PhD, Nancy E. Braverman, MD², \\ Barbara K. Burton, $\mathrm{MD}^{6}$, Dianne M. Frazier, PhD, MPH${ }^{7}$, John Mitchell, MD, Wendy E. Smith, $\mathrm{MD}^{8}$, \\ Barry H. Thompson, MD ${ }^{9}$ and Susan A. Berry, MD ${ }^{10}$; For the American College of Medical Genetics and \\ Genomics Therapeutics Committee
}

\begin{abstract}
Disclaimer: This guideline is designed primarily as an educational resource for clinicians to help them provide quality medical services. Adherence to this guideline is completely voluntary and does not necessarily assure a successful medical outcome. This guideline should not be considered inclusive of all proper procedures and tests or exclusive of other procedures and tests that are reasonably directed to obtaining the same results. In determining the propriety of any specific procedure or test, the clinician should apply his or her own professional judgment to the specific clinical circumstances presented by the individual patient or specimen. Clinicians are encouraged to document the reasons for the use of a particular procedure or test, whether or not it is in conformance with this guideline. Clinicians are also advised to take notice of the date this guideline was adopted and to consider other medical and scientific information that becomes available after that date. It also would be prudent to consider whether intellectual property interests may restrict the performance of certain tests and other procedures.
\end{abstract}

Phenylalanine hydroxylase deficiency, traditionally known as phenylketonuria, results in the accumulation of phenylalanine in the blood of affected individuals and was the first inborn error of metabolism to be identified through population screening. Early identification and treatment prevent the most dramatic clinical sequelae of the disorder, but new neurodevelopmental and psychological problems have emerged in individuals treated from birth. The additional unanticipated recognition of a toxic effect of elevated maternal phenylalanine on fetal development has added to a general call in the field for treatment for life. Two major conferences sponsored by the National Institutes of Health held $>10$ years apart reviewed the state of knowledge in the field of phenylalanine hydroxylase deficiency, but there are no generally accepted recommendations for therapy. The purpose of this guideline is to review the strength of the medical literature relative to the treatment of phenylalanine hydroxylase deficiency and to develop recommendations for diagnosis and therapy of this disorder. Evidence review from the original National Institutes of Health consensus conference and a recent update by the Agency for Healthcare Research and Quality was used to address key questions in the diagnosis and treatment of phenylalanine hydroxylase deficiency by a working group established by the American College of Medical Genetics and Genomics. The group met by phone and in person over the course of a year to review these reports, develop recommendations, and identify key gaps in our knowledge of this disorder.

Phenylalanine hydroxylase (PAH) deficiency, traditionally called phenylketonuria (PKU) due to characteristic phenylketones accumulating in the urine of affected individuals, has a significant place in history as the first inborn error of metabolism identified through population-based screening, initiating
Above all, treatment of phenylalanine hydroxylase deficiency must be life long, with a goal of maintaining blood phenylalanine in the range of $120-360 \mu \mathrm{mol} / \mathrm{l}$. Treatment has predominantly been dietary manipulation, and use of low protein and phenylalanine medical foods is likely to remain a major component of therapy for the immediate future. Pharmacotherapy for phenylalanine hydroxylase deficiency is in early stages with one approved medication (sapropterin, a derivative of the natural cofactor of phenylalanine hydroxylase) and others under development. Eventually, treatment of phenylalanine hydroxylase deficiency will be individualized with multiple medications and alternative medical foods available to tailor therapy. The primary goal of therapy should be to lower blood phenylalanine, and any interventions, including medications, or combination of therapies that help to achieve that goal in an individual, without other negative consequences, should be considered appropriate therapy. Significant evidence gaps remain in our understanding of the optimal therapies for phenylalanine hydroxylase deficiency, nonphenylalanine effects of these therapies, and long-term sequelae of even welltreated disease in children and adults.

Genet Med advance online publication 2 January 2014

Key Words: maternal PKU; phenylalanine hydroxylase deficiency; phenylketonuria; sapropterin; therapy

a new era in the diagnosis and treatment of genetic disorders. PKU was first described in 1934 by the Norwegian physician Asbjörn Fölling, but it was not until the mid-1950s that a patient with PAH deficiency was treated with a low phenylalanine (PHE) diet. Although this first patient already had irreversible

J.V. and S.A.B. contributed equally to this work.

${ }^{1}$ Department of Pediatrics, University of Pittsburgh School of Medicine, Pittsburgh, Pennsylvania, USA; ${ }^{2}$ Department of Human Genetics, University of Pittsburgh Graduate School of Public Health, Pittsburgh, Pennsylvania, USA; ${ }^{3}$ Hayward Genetics Center, Tulane University Medical School, New Orleans, Louisiana, USA; ${ }^{4}$ Department of Psychology, Syracuse University, Syracuse, New York, USA; ${ }^{5}$ Department of Human Genetics and Pediatrics, McGill University, Montreal, Quebec, Canada; ${ }^{6}$ Department of Pediatrics, Northwestern University Medical School, Chicago, Illinois, USA; ${ }^{7}$ Department of Pediatrics, University of North Carolina at Chapel Hill, Chapel Hill, North Carolina, USA; ${ }^{8}$ Department of Pediatrics, Maine Medical Center, Portland, Maine, USA; ${ }^{9}$ The American College of Medical Genetics and Genomics, Bethesda, Maryland, USA; ${ }^{10}$ Department of Pediatrics, University of Minnesota Medical School, Minneapolis, Minnesota, USA. Correspondence: Jerry Vockley (gerard.vockley@chp.edu) 
developmental disabilities, clinical improvement was observed and blood PHE levels declined. At about this time, the underlying biochemical defect, decreased activity of hepatic PAH, was identified. PAH deficiency most accurately describes the spectrum of clinical phenotypes ranging from PKU to hyperphenylalaninemia and thus will be used throughout this guideline. Tetrahydrobiopterin $\left(\mathrm{BH}_{4}\right)$ is a necessary cofactor for PAH activity, and rare genetic defects in the pathway of $\mathrm{BH}_{4}$ synthesis or recycling can lead to secondary PAH deficiency and elevated blood PHE levels. Treatment of these defects, which present differently than PAH deficiency and have largely different therapy, is not considered further in this guideline.

PAH deficiency presents a spectrum of severity, and several different classification schemes have been proposed to assist in clinical management. Most severe are individuals with complete enzyme deficiency whose untreated blood PHE levels are typically $>1,200 \mu \mathrm{mol} / \mathrm{l}$ (mean normal level: $60 \mu \mathrm{mol} / \mathrm{l}$ ); this phenotype is consistently termed "classical PKU." It should be noted that infants diagnosed and treated earlier in life might have a peak PHE level $<1,200$ $\mu \mathrm{mol} / \mathrm{l}$ and still have complete PAH deficiency. In 2000, the National Institutes of Health (NIH) Consensus Development Conference Statement categorized all patients with untreated blood PHE levels greater than normal level but less than 1,200 $\mu \mathrm{mol} / \mathrm{l}$ as having hyperphenylalaninemia. ${ }^{1}$ This guideline recommends a unifying nomenclature and therefore refers to the spectrum of PAH deficiency, not specifically relying on the blood PHE level, although we recognize that the most severe form is still likely to be referred to as "classical PKU" in many settings.

Newborn screening (NBS) for PAH deficiency became widespread in North America and in the United Kingdom by the mid- to late-1960s and in the rest of the developed world by the early 1970s. Since the initiation of NBS, almost all cases of PAH deficiency are diagnosed following a positive newborn screening test, resulting in significant economic savings to society in addition to unquestioned benefits for affected individuals. ${ }^{2,3}$ Dietary therapy involving dietary PHE restriction and supplementation with reduced or PHE-free amino acid mixtures (medical foods, "formulas") is effective in preventing severe mental retardation associated with untreated classical PAH deficiency. Over time, subtle intellectual and neuropsychiatric issues may manifest even with treatment. In addition, patients treated from the early weeks of life with initial good metabolic control, but who lose that control in later childhood or adult life, may experience both reversible and irreversible neuropsychiatric consequences. Even severely intellectually disabled adults with late-diagnosed PAH deficiency show improvements in challenging behavior with lowering of blood PHE levels. ${ }^{4}$ Pregnancy presents a particular problem in women with $\mathrm{PAH}$ deficiency; as high levels of PHE are toxic to the brain of the developing fetus and, along with other teratogenic effects, result in a defined maternal PKU (MPKU) syndrome.

$\mathrm{PAH}$ deficiency is an autosomal-recessive disorder. The gene is located on chromosome 12q23.1. More than 500 different mutations in the PAH gene have been described; ${ }^{5}$ most are point mutations, but deletions, duplications, and insertions are also observed. Most missense mutations result in abnormal folding of the PAH protein, increased protein turnover, and/or decreased activity. Although genotype-phenotype correlations are imperfect, genotype is clearly the best clinically available predictor of severity in PAH deficiency; siblings tend toward similar degrees of enzyme deficiency and dietary PHE tolerance. In compound heterozygotes, the less severe mutation tends to determine the clinical severity of disease. ${ }^{6} \mathrm{PAH}$ deficiency is common in Caucasians in whom the overall incidence is 1 in 10,000 live births: ${ }^{7}$ it is particularly common in Ireland and Turkey where the incidence is 1 in 4,500 and 1 in 2,600, respectively.

\section{METHODS AND PROCESS}

Evidence review for this guideline relied upon two independent review processes described previously. The first was a NIH consensus conference held in 2000. ${ }^{1}$ The second review was performed by the Agency for Healthcare Research and Quality (AHRQ) as a precursor to a more recent NIH conference (March 2012). ${ }^{8}$ Please see the Supplementary Material online for additional details. Key questions addressed by the AHRQ are contrasted with those in the NIH review in Supplementary Table 1 online. To complete the literature review, papers published in MEDLINE between the time of the most recent AHRQ review and the date of the work group meeting (September 2012) were searched for any reference to PKU or phenylketonuria. Eighty additional references were found and reviewed face to face by the working group. The working group worked by teleconference (weekly) to prepare the guideline outline, discuss levels of evidence, and initial recommendations. To assemble the final guideline, working group members met in person to review both of the prior evidence review documents, along with more recent literature abstracted following the AHRQ review. To formulate recommendations, each component of the guideline was discussed individually, and a consensus recommendation regarding these guidelines was made based on agreement of $75 \%$ of the working group.

Level of evidence and recommendations were assigned according to the Scottish Intercollegiate Guideline Network (SIGN; http://www.sign.ac.uk/), an evidence-based protocol for evaluating the medical literature on clinical therapy and trials and grading treatment recommendations based on that literature. Except for the use of sapropterin (where the evidence level is 1 and a recommendation SIGN grade of A could be assigned), available evidence is primarily level 3 or 4 and all recommendations are of grade C or D. Due to the limited utility of such a skewed grading system for our purposes, these grades will not be further emphasized in the remainder of this document. ${ }^{9}$

\section{Newborn screening}

\section{NBS AND DIAGNOSTIC TESTING}

With development of the bacterial inhibition assay for PHE using blood samples collected on filter paper cards for sample 
procurement and transport, it became possible to establish NBS programs for PAH deficiency. ${ }^{10}$ Screening is now done using tandem mass spectrometry (MS/MS). ${ }^{11}$ Elevated PHE concentrations in blood spots can be quantified as early as $24 \mathrm{~h}$ after birth, and tyrosine (TYR) concentrations can be used to calculate a PHE:TYR ratio. The distinction between PAH deficiency and generalized aminoacidemia is made with detection and quantitation of additional amino acids in the screening panel. As part of NBS for multiple inborn errors of metabolism, MS/MS NBS for PAH deficiency has been shown to be cost beneficial. ${ }^{12}$

Although MS/MS-based NBS is far more accurate in determining blood PHE concentration than older screening methods, observation of an elevated PHE level is nonspecific and does not definitively indicate that PAH deficiency is present. Most NBS laboratories determine their own cut-off levels above which a test is considered to be positive and requires further evaluation. An international database of 133 contributing laboratories reports a mean cutoff for PHE of $130 \mu \mathrm{mol} / \mathrm{l}$ (with a range of $65-234 \mu \mathrm{mol} / \mathrm{l}$ ) and a PHE:TYR ratio $>3$ as abnormal. ${ }^{13}$ Elevated levels should trigger further evaluation, and assessment should include tests to identify defects in $\mathrm{BH}_{4}$ synthesis or regeneration. Further guidance on follow-up of an abnormal NBS test for PHE can be found in the ACMG ACT sheets for newborns along with confirmatory testing algorithms (www. acmg.net).

\section{Diagnostic testing}

Plasma amino acid analysis is the standard method for confirming elevated PHE in newborns having a positive NBS. Samples are typically taken before dietary PHE restriction is initiated. The analysis should quantify PHE, PHE:TYR ratio and complete amino acid profile. As it may take several days before the NBS results are known, newborns who have PAH deficiency typically have plasma PHE concentrations on confirmatory testing that are increased over the original sample and greater than the upper limit of normal for newborn plasma without any indication of generalized aminoacidemia. ${ }^{14}$

\section{Cofactor deficiency testing}

Disorders of $\mathrm{BH}_{4}$ synthesis and regeneration should be evaluated in all newborns with elevated PHE on NBS. ${ }^{15}$ Pterins must be measured in urine or blood. ${ }^{16}$ As this will not detect all disorders of tetrahydrobiopterin metabolism, erythrocyte dihydropterin reductase should be measured on whole blood spotted on filter paper. A quantitative assay for urinary neopterin and biopterin can confirm results obtained from the filter paper samples. ${ }^{15}$ Reference values are available for different age groups. ${ }^{17}$ Abnormal pterin levels and ratios should prompt enzyme testing for possible deficiencies of: GTP cyclohydrolase, 1,6-pyruvoyl-tetrahydrobiopterin synthetase, dihydropteridine reductase, or pterin carbinolamine- $4 a$-dehydratase. In addition, there are other defects in the pterin generation/regeneration pathway that do not manifest with elevated PHE and are not detected by NBS as currently performed.

\section{PAH activity}

Enzymatic activity for PAH is detectable in hepatic and renal tissues only and is not appropriate for either screening or diagnostic testing.

\section{Genotyping}

The human PAH gene is located at chromosome 12q23.1, spanning $\sim 100 \mathrm{~kb}$ and is composed of 13 exons. Only about $25 \%$ of the human $\mathrm{PAH}$ genotypes are homoallelic, which makes genotype/phenotype correlations difficult. Over 600 mutations have been described (see http://www.ncbi.nlm.nih.gov/ books/NBK1504/). PAH deficiency is a multifactorial disorder requiring both exposure to dietary $\mathrm{PHE}$ and genetic deficiency of PAH activity. Individuals with similar mutant $\mathrm{PAH}$ genotypes may have disparate phenotypes. That said, an individual's specific PAH genotype is still the major determinant of metabolic phenotype. ${ }^{18,19}$ Mutation analysis should be obtained for all infants with elevated PHE to provide information that may affect the extent of dietary PHE restriction and the likelihood of response to cofactor $\left(\mathrm{BH}_{4}\right.$; sapropterin) supplementation, with submission of results to the $\mathrm{PAH}$ databases. ${ }^{20-23}$

\section{KEY POINTS}

- NBS for PAH deficiency in the United States is now primarily performed by tandem mass spectrometry.

\section{RECOMMENDATIONS}

- Quantitative blood amino acids should be performed as part of the diagnostic testing for follow-up of a positive NBS;

- Additional testing is needed to define the cause of elevated blood PHE and should include analysis of pterin metabolism;

- PAH genotyping is indicated for improved therapy planning.

\section{INITIATION OF THERAPY}

Initiation of treatment for PKU should be undertaken as early as possible, preferably within the first week of life with a goal of having blood PHE in the treatment range within the first 2 weeks of life. Upon diagnosis, blood PHE level should be lowered into the desired treatment range as quickly as possible. Depending on the initial blood PHE levels, PHE may be excluded from the diet until levels approach the treatment range followed by initiation and titration of a PHE-restricted diet. ${ }^{1}$ Breastfeeding is often possible in combination with medical formula. Early initiation of treatment requires prompt NBS, follow-up, and diagnostic testing; open communication between the family and the primary care provider; and access to appropriate specialty care.

Infants whose blood PHE levels exceed $600 \mu \mathrm{mol} / 1$ require treatment. ${ }^{24}$ Many treatment centers in North America now initiate treatment at a PHE level of $360 \mu \mathrm{mol} / \mathrm{l}$ or higher; however, the evidence regarding clinical outcome in untreated patients with blood PHE levels between 360 and $600 \mu \mathrm{mol} / \mathrm{l}$ is mixed, with some studies demonstrating normal outcomes and others showing subtle neurocognitive deficits. ${ }^{25-28}$ More research is needed 
to inform decisions regarding treatment of individuals with PHE levels in this category. Given the potential risk for neurocognitive consequences, treatment of infants with sustained blood PHE levels $>360 \mu \mathrm{mol} / \mathrm{l}$ is recommended following appropriate review of the controversy with parents. While a threshold for the adverse effects of elevated blood PHE has not been proven, treatment for infants with PHE levels between 120 and $360 \mu \mathrm{mol} / \mathrm{l}$ is not recommended, although these individuals should be followed for the first 2 years of life (at minimum) to ensure that levels do not drift upward with higher protein intake. If treatment is not required before 2 years of age, monitoring on an annual or biennial basis is adequate for subsequent assessment.

\section{DIETARY THERAPY}

\section{Nutrient requirements in PAH deficiency}

Dietary therapy with restriction of dietary PHE intake remains the mainstay of therapy for PAH deficiency, requiring a decrease in the intake of natural protein and replacing it with a protein (amino acid mixture) source devoid of PHE. An experienced metabolic physician and nutritionist team should manage this therapy. Infants newly diagnosed with PAH deficiency should be followed in clinic and have blood levels monitored frequently until PHE levels have stabilized. Because foods normally consumed as protein sources contain other essential nutrients, it is important that a diet modified for PAH deficiency provides sources for all other nutrients necessary for normal growth and health maintenance. Detailed dietary management of PAH deficiency is described in the accompanying nutrition recommendations authored by the Genetic Metabolic Dietitians International and Southeast Regional Newborn Screening and Genetics Collaborative..$^{29}$ The dietary reference intakes for the general population are modified for individuals with PAH deficiency by adding specific recommendations for PHE, TYR, and protein (Table 1; see companion paper from the Genetic Metabolic Dietitians International/Southeast Regional Newborn Screening and Genetics Collaborative for references). The wide ranges given for PHE intake reflect the influence of many factors on PHE requirements, including residual PAH deficiency activity, patient age, growth rate, sapropterin-responsiveness, etc. In all cases, blood PHE level is the final determinant of modification of dietary PHE intake.

\section{Medical foods for PAH deficiency}

Except in the mildest forms of PAH deficiency, the degree of PHE (and protein) restriction in a natural diet altered to achieve lowered blood PHE levels will result in inadequate protein to support normal growth and health unless a PHEfree, amino acid-based medical food is consumed to supplement protein, calories, and other nutrients. Low-proteinmodified foods and PHE-free medical food beverages are medically necessary for patients with $\mathrm{PAH}$ deficiency and should be regarded as medications. Products containing a PHE-free amino acid mixture have long been the mainstay of dietary therapy for PAH deficiency and are designed to meet established dietary requirements, as well as cater
Table 1 GMDI/SERC recommended intakes of PHE, TYR, and protein for individuals with PAH deficiency

\begin{tabular}{|c|c|c|c|}
\hline Age & $\begin{array}{c}\text { PHE } \\
\text { (mg/day) }\end{array}$ & $\begin{array}{c}\text { TYR } \\
\text { (mg/day) }\end{array}$ & $\begin{array}{c}\text { Protein }^{a} \\
(\mathrm{~g} / \mathrm{kg})\end{array}$ \\
\hline \multicolumn{4}{|l|}{ Infants to $<4$ year ${ }^{b}$} \\
\hline 0 to $<3$ months ${ }^{c}$ & $130-430$ & $1,100-1,300$ & $3-3.5$ \\
\hline 3 to $<6$ months & $135-400$ & $1,400-2,100$ & $3-3.5$ \\
\hline 6 to $<9$ months & $145-370$ & $2,500-3,000$ & $2.5-3$ \\
\hline 9 to $<12$ months & $135-330$ & $2,500-3,000$ & $2.5-3$ \\
\hline 1 to $<4$ years $^{d}$ & $200-320$ & $2,800-3,500$ & $\geq 30$ \\
\hline \multicolumn{4}{|l|}{4 years to adulte } \\
\hline 4 years to adult & $200-1,100^{\mathrm{e}}$ & $4,000-6,000$ & $\begin{array}{l}120-140 \% \\
\text { RDA for age }\end{array}$ \\
\hline \multicolumn{4}{|l|}{ Pregnancy/lactation ${ }^{9}$} \\
\hline Trimester 1 & $265-770$ & $6,000-7,600$ & $\geq 70$ \\
\hline Trimester 2 & $400-1,650$ & $6,000-7,600$ & $\geq 70$ \\
\hline Trimester 3 & $700-2,275$ & $6,000-7,600$ & $\geq 70$ \\
\hline Lactation ${ }^{h}$ & $700-2,275$ & $6,000-7,600$ & $\geq 70$ \\
\hline
\end{tabular}

DRI, dietary reference intakes; GMDI, Genetic Metabolic Dietitians International; PAH, phenylalanine hydroxylase; PHE, phenylalanine; PKU, phenylketonuria; RDA, required daily allowance; SERC, Southeast Regional Newborn Screening and Genetics Collaborative.

aProtein recommendations for individuals consuming PHE-free amino acid-based medical foods as part of their protein source. ${ }^{b}$ Recommended intakes for infants and children $<4$ years of age are for individuals with a severe form of PAH deficiency treated with a PHE-restricted diet alone. Recommendations for energy and fluid intakes do not differ from normal population. CPHE requirements for premature infants

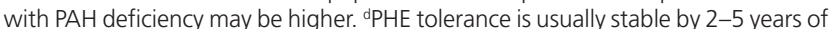
age as PHE requirements are based on a combination of size (increasing with age) and rate of growth (decreasing with age). For any individual PHE intake is adjusted based

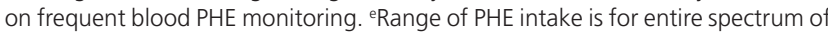

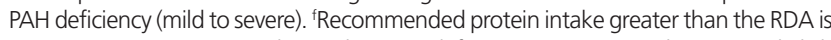

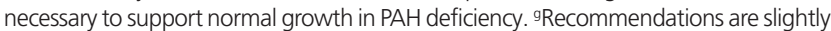
higher for pregnant women $\leq 19$ years of age. " $D R I$ recommend that, for all women, nutrient intake during lactation be the same as during the third trimester of pregnancy. From Ref 29.

to individual needs and preferences (i.e., taste and consistency). Glycomacropeptide, a natural by-product of cheese production, has a very low PHE content so several medical food products have recently been developed using it as a protein source. ${ }^{30}$ The availability of a variety of medical food products gives patients and their providers many options to facilitate compliance with dietary PHE restriction; however, medical food choices may also impact nutritional status, reinforcing the need for careful monitoring.

Modified low-protein foods are another category of medical foods important to PAH-deficiency diet management as an important source of calories and to provide satiety to patients on low protein and PHE-restricted meals. Many of these foods, such as baked goods and pastas, are prepared with starches from wheat and other grains to reduce the protein (and PHE) content normally derived from flour. Modified low-protein foods that mimic higher protein foods help to increase dietary variety and help to normalize the look of the low PHE diet. Access to these low protein foods is a challenge as they are more costly 
than their higher protein, nonmodified counterparts and this cost may not always be covered by third-party payers.

\section{Goals and monitoring of therapy}

Dietary manipulation required to maintain appropriate blood PHE levels needs frequent modification to respond to growth, life stages, concurrent illness, and comorbidities. It is important to monitor blood PHE and TYR levels and to ensure that other nutritional requirements are also being met. ${ }^{31}$ Essential fatty acid and vitamin/mineral status is of special concern in patients consuming medical foods that may not contain appropriate amounts for their individual circumstances.

Blood PHE levels in all patients should be maintained in the range of $120-360 \mu \mathrm{mol} / \mathrm{l}$. At present, there is no evidence to suggest that normalization of blood PHE levels is required, but levels in the range of $60-120 \mu \mathrm{mol} / \mathrm{l}$ should not be regarded as "too low," particularly in the patient whose PHE intake is not severely restricted. Adequate monitoring of blood PHE levels in such patients should eliminate any potential risk associated with prolonged low $(<30 \mu \mathrm{mol} / \mathrm{l})$ blood PHE levels. The measure of PHE levels in blood varies dependent on the analytical method used; therefore, consistency of testing methodology is important for comparison over time. ${ }^{32}$ Newly diagnosed infants should be seen in clinic frequently with PHE and TYR testing until the PHE levels are stabilized. Subsequently, blood PHE levels should be monitored at least weekly until age 1 with increased surveillance during periods of rapid growth and transitions of diet, such as with the introduction of solid foods. After 1 year of age and until 12 years of age, biweekly to monthly sampling is often adequate. In adolescents and adults who are stable and well controlled, monthly testing may be adequate. In addition, plasma amino acids (full panel), transthyretin, albumin, complete blood count, ferritin, 25-OH vitamin $\mathrm{D}$, vitamin $\mathrm{B}_{12}$, red blood cell essential fatty acids, trace minerals (zinc, copper, and selenium) vitamin $\mathrm{A}$, comprehensive metabolic panel, and folic acid testing should be considered when a formal nutritional assessment suggests suboptimal dietary intake or over-reliance on nutritionally incomplete medical foods. Bone mineralization may be suboptimal due to the low intake of calcium from natural dairy sources in a protein-restricted diet. The utility of routine DEXA scans to monitor bone density has not yet been established. ${ }^{33}$

\section{PHARMACOTHERAPY}

In 2007, the first pharmacologic agent for the treatment of PAH deficiency, sapropterin dihydrochloride, was approved by the US Food and Drug Administration. Sapropterin (Kuvan, BioMarin Pharmaceutical, Novato, CA) is a synthetic form of the naturally occurring cofactor, tetrahydrobiopterin. ${ }^{19,34}$ Although not deficient in endogenous tetrahydrobiopterin, some patients with PAH deficiency who have some residual enzyme activity respond to administration of sapropterin with an increase in the metabolism of PHE to TYR. The mechanism by which residual $\mathrm{PAH}$ activity enhanced is unclear, but $\mathrm{BH}_{4}$ may act as a pharmacologic chaperone leading to improved folding and increased stability of the mutant protein. Approximately $25-50 \%$ of the patients with PAH deficiency are sapropterin-responsive. ${ }^{19,35-37}$ Patients with mild PAH deficiency are most likely to respond because some stable protein is required for sapropterin to function; nonetheless, responsive patients are identified even among those with complete PAH deficiency. Genotype may be predictive of sapropterin response, but genotype-phenotype correlations thus far are imperfect. ${ }^{19}$ Therefore, every PAH-deficient patient should be offered a trial of sapropterin therapy to assess responsiveness except those with two null mutations in trans.

Sapropterin is given typically once a day at a dose of $5-20 \mathrm{mg} /$ $\mathrm{kg}$. The most commonly used dose used for initiation and maintenance is $20 \mathrm{mg} / \mathrm{kg}^{38,39}$ During clinical trials, no serious side effects of sapropterin were identified. ${ }^{19,34}$ Before routine treatment with sapropterin is initiated, a test should be conducted to determine if the patient is responsive. ${ }^{39}$ While testing is typically done in Europe at the time of initial diagnosis, this is not the practice in the United States. If testing is to be done in early infancy, it is recommended that blood PHE levels first be lowered to $480-600 \mu \mathrm{mol} / \mathrm{l} .{ }^{16}$ Sapropterin has been used under the age of 4 years in PAH deficiency and in patients with biopterin synthesis defects. ${ }^{36,40}$ Sapropterin responsiveness is commonly determined by obtaining a baseline blood PHE level on the day the medication is initiated (baseline) and then starting the patient on a single daily dose of sapropterin at $20 \mathrm{mg} / \mathrm{kg}$. Additional blood PHE levels are then obtained at regular intervals, usually at $24 \mathrm{~h}, 1$ week, 2 weeks, and in some cases, 3 or 4 weeks. Testing at doses $<20 \mathrm{mg} /$ $\mathrm{kg}$ underestimates the response rate and is not recommended. A significant decline in blood PHE is expected in responders once treatment is initiated with the assumption that the diet remains stable throughout the testing period. Clinical judgment is required to determine what constitutes a significant or beneficial decline in an individual patient, but $30 \%$ is often cited in the literature as evidence of effective PHE reduction. Most sapropterinresponsive patients have a rapid decline in blood PHE level, but occasionally a delay of $2-4$ weeks is seen. Patients with a baseline PHE level at the lower end of the treatment range $(180 \mu \mathrm{mol} / \mathrm{l}$ or lower) rarely show a significant decline in blood PHE level, even if they are sapropterin-responsive. In these patients, responsiveness must be determined by adding additional PHE to the diet in a stepwise fashion to determine if an increase in PHE tolerance is achieved, i.e., maintenance of low blood PHE levels on increased dietary PHE. ${ }^{41}$ An improvement in neuropsychiatric symptoms or increase in PHE tolerance without a decrease in blood PHE in any patient constitutes sufficient justification to continue therapy. For patients who are able to maintain blood PHE levels in the desired range with dietary treatment alone, the major benefit of treatment with sapropterin is that it increases dietary protein and PHE tolerance in responsive patients, allowing for the inclusion of more natural protein in the diet. In some patients, the increase in dietary PHE can be substantial (two- to threefold over baseline) and has an impact on quality of life. For most sapropterin-responsive patients, the benefits of treatment are such that long-term therapy with sapropterin should be maintained.

Large neutral amino acids (LNAA) have been proposed as a therapy for PAH deficiency based on their ability to block uptake 
of PHE (which is a large neutral amino acid) from the intestine and at the blood-brain barrier. A single clinical trial demonstrated reduction of blood PHE by $\sim 40 \%$ following substitution of a standard low PHE medical food with one supplemented with LNAA at a dose of 0.5 or $1.0 \mathrm{~g} / \mathrm{kg}$ of body weight. ${ }^{42}$ Larger trials are necessary to substantiate this finding, to demonstrate an effect on symptoms unrelated to PHE level, and to determine long-term safety. Treatment with LNAA is currently limited to older patients (adolescents and adults) and should be avoided in pregnant women due to a limited understanding of their effects on fetal growth and the developing central nervous system of the fetus. Furthermore, LNAA are contraindicated as monotherapy in pregnant women because LNAA therapy does not sufficiently lower blood PHE to the range that is safe for fetal development.

It is likely that other pharmacologic agents will ultimately become available to treat PKU. The compound closest to clinical use is polyethyleneglycol-conjugated phenylalanine ammonia lyase (PEG-PAL), which entered phase III clinical trials in 2013. It appears to be effective in lowering blood PHE levels, even in patients on a completely unrestricted diet. It is administered as a daily subcutaneous injection and metabolizes blood PHE by a mechanism independent of PAH and so would be theoretically effective in any patient with $\mathrm{PAH}$ deficiency. ${ }^{43}$ Immunologic reactions were reported in the phase II trial. Phenylalanine ammonia lyase is a bacterial enzyme that degrades PHE to transcinnamic acid; trans-cinnamic acid and its final product, benzoic acid, are conjugated with glycine and excreted in the urine.

Eventually, treatment of PAH deficiency will be individualized with multiple medications and alternative medical foods available to tailor therapy. The primary goal of therapy should be to lower blood PHE, and any interventions, including medications, or combination of therapies that help to achieve that goal in an individual, without other negative consequences, should be considered appropriate therapy. Secondary goals should include improved dietary PHE tolerance, amelioration of symptoms, and improved quality of life. Effects of adjuvant therapies on PHE tolerance require continued careful clinical and laboratory monitoring.

\section{KEY POINTS}

- Sapropterin is currently the only FDA-approved medication for the treatment of PAH deficiency and may be useful in reducing PHE levels in responsive patients;

- Experience with sapropterin under the age of 4 years is limited;

- Response to sapropterin is not accurately predicted by genotype and thus should be documented by formal testing

\section{RECOMMENDATIONS}

- Any combination of therapies that facilitate improvement in blood PHE levels for a given individual is appropriate; therapies may be combined and should be individualized;

- Reduction of blood PHE, increase in dietary PHE tolerance, or improvement in clinical symptoms are all valid indications for continuation of therapy.

\section{TREATMENT FOR LIFE}

There is strong evidence to support the position that treatment and maintenance of metabolic control throughout life is essential to optimal functioning of individuals with PAH deficiency. While intellectual disability does not occur in patients who are well controlled in infancy and childhood, a variety of adverse neurocognitive and psychiatric outcomes, including deficits in executive functioning and psychiatric symptoms such as anxiety, depression, and phobias can develop later in life when there is relaxation of PHE control. ${ }^{44-49}$ These later effects can be disabling and can result in a lower overall level of educational attainment and socioeconomic status..$^{50,51}$ Furthermore, these symptoms make it increasingly difficult for a patient with PAH deficiency to return to metabolic control, since adherence to treatment requires tasks such as planning and organization that rely on intact executive functioning abilities. Therefore, it is recommended that patients be maintained in metabolic control as they move into adulthood. As the clinical spectrum of PAH deficiency is broad, and the needs of patients vary considerably throughout life, it is essential that treatment be tailored to the individual patient.

Historically, liberalization of the PHE-restricted diet and relaxation of PHE control was allowed. As more information has accumulated regarding effects of elevated PHE levels on brain function, this practice is no longer acceptable. ${ }^{52}$ This guideline recommends that blood PHE levels be maintained in the range of $120-360 \mu \mathrm{mol} / \mathrm{l}$ in patients of all ages. While published evidence of harm associated with treated PHE levels in the range of 360-600 $\mu \mathrm{mol} / \mathrm{l}$ has been inconsistent, there is no convincing evidence that these levels are without clinical effect. It is also unknown if adults with PHE levels of 360-600 $\mu \mathrm{mol} / \mathrm{l}$ without $\mathrm{PHE}$ restriction require therapy. With improvements in the treatment of PAH deficiency, and more therapeutic options emerging to facilitate reduction in blood PHE levels, the goal should be sustained blood PHE control for the next generation of patients with this condition.

Patients with PAH deficiency who were treated in early life but in whom treatment has ceased represent a major therapeutic challenge. Many of these patients no longer have contact with a treatment clinic, and some are unaware that they have a disorder for which lifelong treatment is recommended. Affected individuals may have a limited understanding of the nature of PAH deficiency and lack knowledge of advances in dietary treatment and availability of pharmacologic therapy. They may also have significant neurocognitive deficits and be unaware that these issues are related to their diagnosis of PAH deficiency. This guideline recommends that clinics make every effort to contact these "out-of-clinic" patients and encourage their return to treatment.

Patients with late-treated PAH deficiency and severe cognitive impairment also represent a special challenge. While it is unlikely that there will be improvement in cognitive abilities even if dietary treatment successfully reduces blood PHE, there is anecdotal evidence suggesting that patients may exhibit improvements in behavior, psychiatric symptomatology, and 
seizure control. ${ }^{4}$ Therefore, a trial of treatment is justified in such patients. If desired, a sapropterin response test can be conducted while the patient is on an unrestricted diet. If no response is demonstrated, or if dietary interventions are preferred, stepwise dietary changes should be made until the blood PHE level is brought under control. Treatment for a minimum of 6 months is recommended with blood PHE monitoring and careful observation by family members or caretakers. Discontinuation can be considered if no positive benefits are demonstrated after that time.

Girls and women in whom no treatment has been initiated but who have persistent blood PHE levels between 360 and 600 $\mu \mathrm{mol} / \mathrm{l}$ should receive continued monitoring and education, as they will require treatment prior to and during pregnancy. Ongoing interaction with clinics is recommended to reduce attrition and to facilitate initiation of diet and medical food in anticipation of pregnancy.

\section{KEY POINTS}

- Patients who have discontinued therapy will likely experience neuropsychological improvements with reinstitution of therapy;

- Patients with late or untreated PAH deficiency may benefit from institution of therapy.

\section{RECOMMENDATIONS}

- Treatment for PAH deficiency should be lifelong for patients with untreated PHE levels >360 $\mu \mathrm{mol} / /$;

- Maintaining a treated PHE level of 120-360 $\mu \mathrm{mol} / \mathrm{l}$ is recommended for all patients of all ages.

\section{MATERNAL PAH DEFICIENCY}

The success of NBS for PAH deficiency leading to improved outcomes has led to an increasing number of women with this disorder bearing children. The teratogenic effects of PHE on the developing fetus, termed MPKU syndrome, refers to the physical and cognitive effects on the fetus of in utero exposure to elevated PHE levels including microcephaly, poor fetal growth, congenital heart defects (CHD), nonfamilial facial features, and intellectual disability. ${ }^{53}$ Since the identification of MPKU syndrome, concerns have been raised regarding the degree to which inadequate maternal treatment could negate the positive societal and economic effects of early identification through NBS. $^{53}$ Approximately $65 \%$ of PAH-deficient mothers have poorly controlled PHE before 8 weeks gestation. ${ }^{54}$

\section{Clinical features}

Intellectual disability is the most consistent feature of the MPKU syndrome, occurring in $>90 \%$ of the children born to women who never achieve PHE control during their pregnancy. Data from the MPKU study compiled by the AHRQ identified a threshold of maternal PHE at $360 \mu \mathrm{mol} / \mathrm{l}$, above which there is a linear relationship between maternal PHE level and decreasing cognitive outcomes in offspring. There is emerging evidence of adverse behavioral outcomes, including externalizing behaviors, in children born to women with suboptimal PHE control during pregnancy. ${ }^{55}$ Microcephaly is the most common fetal malformation associated with elevated maternal PHE levels during gestation. The incidence of microcephaly increases to $5-18 \%$ with achievement of control by 10 weeks gestation and steadily increases to $67 \%$ if PHE control is not achieved by 30 weeks gestation. ${ }^{56}$ The increased risk of CHD is related to the timing of fetal cardiac development and maternal PHE control. As the fetal heart develops prior to 8-10 weeks of gestation, consistently elevated maternal PHE ( $>600 \mu \mathrm{mol} / \mathrm{l})$ during early gestation is associated with an increased risk of malformations of between 8 and 12\%. Risks for CHD may also be increased in individuals due to poor protein intake and vitamin $B_{12}$ deficiency ${ }^{56}$ The incidence of intrauterine growth retardation is not increased if PHE control is achieved by 10 weeks gestation; however it increases with later onset of PHE control. ${ }^{57}$

Additional attention should be paid to women with PAH deficiency who have themselves had poor PHE control throughout their lives. Lower maternal IQ, $<85$, is associated with later achievement of gestational PHE control and poorer fetal outcomes, although when optimal PHE control is achieved in mothers irrespective of their IQ, the fetal outcomes are improved..$^{57}$

\section{KEY POINTS}

- Fetal development is optimal when maternal PHE levels are $<360 \mu \mathrm{mol} / \mathrm{l}$ prior to conception;

- There is a linear relationship between maternal PHE levels $>360 \mu \mathrm{mol} / \mathrm{l}$ throughout gestation and lower IQ of the developing fetus;

- Elevated blood PHE levels in the first 8-10 weeks of gestation are associated with an increased risk of CHD and poor fetal growth.

\section{RECOMMENDATIONS}

- Achievement of maternal PHE levels $<360 \mu \mathrm{mol} / \mathrm{l}$ prior to conception is recommended.

\section{Management during pregnancy}

PHE is transported across the placenta and results in fetal levels that are higher than maternal blood levels. Current data support a goal for maternal PHE levels of 60-360 $\mu \mathrm{mol} / \mathrm{l}$, though international recommendations recommend maintenance of PHE $<240 \mu \mathrm{mol} / 1 .{ }^{58}$ Although studies have shown that PHE levels as low as $100 \mu \mathrm{mol} / \mathrm{l}$ are safe during gestation, there are concerns that persistently low maternal PHE levels, especially during the second and third trimesters, may be associated with an increased risk of IUGR. ${ }^{57}$ The adverse effects of elevated PHE on the developing fetus warrant increased attention and intervention during gestation, with emphasis on preconception control as optimal. Women who become pregnant without appropriate PHE control will need significant support to attain PHE levels within the 
recommended therapeutic range in a timely fashion. This may require intensive intervention, including hospitalization, to initiate dietary control. Not all medications and dietary supplements available for use in individuals with PAH deficiency are suitable for use during pregnancy. Specifically, LNAA should not be used during pregnancy, as they do not consistently alter maternal blood PHE levels. Sapropterin is a class $\mathrm{C}$ medication and may be used during pregnancy when the effects of not using it outweigh its potential adverse effects. There is no evidence of sapropterin-associated teratogenicity or adverse pregnancy effects, and anecdotal reports of successful pregnancy outcomes with its use are increasing. Given the known adverse effects of elevated maternal PHE on pregnancy outcomes, it is the recommendation of this guideline that women taking sapropterin who become pregnant should be offered the option of remaining on the medication, and women who may benefit from sapropterin be offered the option of using it during pregnancy. Ideally, sapropterin response should be determined prior to pregnancy to avoid fluctuations in PHE levels or difficulty in interpretation of responsiveness. Longitudinal data on pregnancy outcomes with and without sapropterin use should be collected (AHRQ Report). ${ }^{8}$

All routine prenatal care is recommended for women with PAH deficiency. Elevated maternal PHE levels do not affect values for routine maternal serum screening testing for other conditions. Fetal growth should be monitored throughout gestation. Early pregnancy dating using ultrasound is recommended due to concerns about later development of intrauterine growth slowing and the possibility of microcephaly. A screening ultrasound for fetal anomalies is also recommended. Fetal echocardiography should be performed at 18-22 weeks gestation. Maternal PHE requirements change significantly throughout gestation necessitating frequent testing and diet adjustments. Dietary over-restriction should be avoided as inadequate protein and calorie intake can contribute to increased maternal PHE levels. Abnormal TYR levels have not been associated with adverse infant outcomes. ${ }^{59}$ Vitamin and mineral intake should be monitored as standard prenatal vitamins taken with medical foods for $\mathrm{PAH}$-deficiency treatment may provide excessive vitamin A, which is associated with birth defects. Decreased intake of vitamin $\mathrm{B}_{12}$ may contribute to an increased risk of CHD. ${ }^{56}$ Please see the accompanying Genetic Metabolic Dietitians International document for details on available supplements and recommended monitoring. ${ }^{29}$

Postpartum maternal PHE requirements will decrease from the increased anabolic requirements of the third trimester, and careful metabolic and nutritional monitoring should continue. Use of medical food may provide the increased calories and protein required to support breastfeeding $(640 \mathrm{kcal} /$ day and $25 \mathrm{~g}$ protein/day). There are no contraindications to breastfeeding, as infants unaffected by PAH deficiency are able to metabolize the slightly higher PHE levels in their mother's breast milk without difficulty..$^{60,61}$

\section{RECOMMENDATIONS}

- LNAA are not recommended for use during pregnancy;

- Sapropterin is a class C medication and may be used during pregnancy following discussion of the benefit and risks to mother and fetus;

- Routine prenatal care and monitoring should be supplemented by close monitoring of fetal growth and assessment for fetal CHD by a high-risk obstetrics group;

- Mothers with PAH deficiency may safely breastfeed;

- Mothers with PAH deficiency should maintain a PHErestricted diet, including use of medical foods, postpartum for optimal maternal/infant outcomes.

\section{GENETIC COUNSELING}

PAH deficiency is inherited in an autosomal-recessive manner; affected individuals inherit two mutations in the $P A H$ gene, one from each parent. There is a $25 \%$ recurrence risk for an affected offspring of carrier parents and a carrier risk of $2 / 3$ for each unaffected full sibling. There are ethnic differences in the population frequency of PAH deficiency, and calculated carrier risk estimates are available for several specific populations. ${ }^{7}$ Because of the dynamic nature of families and emotional, developmental, and psychological issues common in individuals with $\mathrm{PAH}$ deficiency, genetic counseling should be an ongoing process throughout the lifespan.

\section{Carrier testing}

Identification of carriers for PAH deficiency (carrier testing) is most accurate when the genotype of the affected family member is known and targeted testing for the known familial mutation is performed. This can provide close relatives with a clear indication of whether they have an increased risk of being a carrier. Targeted mutation analysis will not detect other mutations in PAH. Population-based carrier testing is not widely available, although several more common PAH-deficiency alleles are included in at least one of the emerging Universal Genetic Testing panels that are commercially available. ${ }^{62}$ Genetic counseling is important to adequately inform couples of their residual risk.

\section{Prenatal testing}

Prenatal diagnosis for PAH deficiency is only available using DNA-based methodologies. Consideration should be given to preconception and prenatal screening for PAH deficiency (obtaining a blood PHE level) for women who have already had one or more children with features suggestive of MPKU syndrome, who have immigrated from countries without universal NBS, who were born prior to the initiation of universal NBS in their countries, or for women with unexplained intellectual disabilities. Determination of fasting plasma or whole blood PHE and TYR concentrations is widely available; interpretation of results can depend on maternal factors such as menstrual cycle timing, contraceptive medication use, and gestational age. ${ }^{63}$ 


\section{KEY POINTS}

- PAH deficiency is inherited in an autosomal recessive manner;

- DNA-based carrier testing of at-risk family members is available;

- There are ethnic differences in the population incidence and carrier frequency of PAH deficiency;

- Prenatal diagnosis for PAH deficiency is only available using DNA-based methodologies.

\section{RECOMMENDATIONS}

- Genetic counseling should be provided as an ongoing process for individuals with PAH deficiency and their families.

\section{NEUROCOGNITIVE AND PSYCHOLOGICAL OUTCOMES}

\section{Intelligence}

Untreated PAH deficiency is associated with intellectual disability; ${ }^{64}$ however, with advances in NBS and treatment, untreated PAH deficiency is rare. Nonetheless, much data have been published indicating an inverse relationship between PHE levels and IQ. ${ }^{64}$ IQ scores in individuals with PAH deficiency are principally related to age of the individual at the time of diet initiation and discontinuation. ${ }^{65}$ Over the past half-century, IQ has served as one of the primary outcome variables for individuals with PAH deficiency, not only for monitoring cognitive development but also for assessing response to treatment. ${ }^{65}$ In the $2000 \mathrm{NIH}$ Consensus Development Conference for PKU, an inverse relationship between IQ and PHE levels was described; however, no specific recommendations were made about how to employ IQ to monitor development or assess response to treatment. ${ }^{1}$ Nonetheless, the recommendation of metabolic control for life by the 2000 Consensus Panel was based on the data demonstrating a decline in IQ scores with relaxation of dietary management. ${ }^{66-68}$

Much literature on early and continuously-treated $\mathrm{PAH}$ deficiency reports IQ scores in the average range; however, pediatric data suggest that even under these circumstances, children with PAH deficiency attain IQ scores that are six to nine points lower than their siblings and parents. ${ }^{69,70}$ Even in a non-PAH-deficient population, IQ scores only partially correlate with school grades and academic achievement, ${ }^{71}$ and therefore further assessment beyond IQ testing is needed for full definition of functional outcomes of management and treatment. ${ }^{69}$ Notably, although studies have not been uniform, deficits in executive function in individuals with PAH deficiency are consistent enough to warrant special attention. ${ }^{27,72-83}$

\section{Psychological issues}

While many individuals with PAH deficiency do not receive psychiatric disorder diagnoses, elevated rates of psychiatric symptoms, especially anxiety and depression, are common. ${ }^{65,66,70,84-86}$ Symptom severity correlates with elevation in PHE, and reduction of PHE generally results in symptom improvement. Numerous studies suggest an increased prevalence of attention problems and attention deficit-hyperactivity disorder in $\mathrm{PAH}$ deficiency; as many as $25 \%$ of the children with early-treated PAH deficiency receive stimulant medication for treating attention deficit-hyperactivity disorder as compared with $7 \%$ of the children with diabetes. There is a need to better characterize the prevalence of attention deficit-hyperactivity disorder and other psychiatric disorders in $\mathrm{PAH}$ deficiency and to assess the response of $\mathrm{PAH}$ patients to standard pharmacological or behavioral interventions. ${ }^{87-89}$ Additional negative effects have emerged in individuals affected with PAH deficiency and their families who have been followed long term. These include consequences of a more rigid style of parenting, delayed autonomy for affected adults, and difficulties forming adult relationships. ${ }^{64,90}$ Despite these concerns, early and continuously treated patients with PAH deficiency can have a normal health-related quality of life. ${ }^{24}$

\section{Recommendations for psychological testing}

Due to the increased risk for neurocognitive and psychological issues in patients with $\mathrm{PAH}$ deficiency, regular mental health monitoring is warranted. Based on a consensus panel of psychologists, a number of psychological tests are recommended as screening tools to identify those in need of further assessment. ${ }^{91}$ Follow-up testing should be directed by screening test results. See Table 2 for recommendations.

\section{KEY POINTS}

- Intelligence in patients with optimally treated PAH deficiency is in the normal range but lower than that in sib controls;

- The incidence of nonintellectual psychological symptoms, especially impairment in executive function, is increased in PAH deficiency.

\section{RECOMMENDATIONS}

- The risk for neurocognitive or psychological symptoms in $\mathrm{PAH}$ deficiency is related to age of onset of therapy, lifelong PHE levels, and adherence to treatment. Age-specific neuropsychiatric and cognitive testing is necessary to adequately assess clinical needs;

- Appropriate intellectual and mental health assessments are an important component of care for individuals affected with PKU.

\section{TRANSITION TO ADULTHOOD}

Transition to adulthood is a high-risk period for individuals with PAH deficiency. As for all adolescents, teens with $\mathrm{PAH}$ deficiency are faced with challenges in maturation including a desire for increased independence, peer pressure, and rebellious behavior. These difficulties are augmented by the necessity of dietary therapy for their chronic disease. When the individual is then asked to leave their trusted pediatric medical environment and enter an adult setting, there is a risk for loss of metabolic control. These patients are also at high risk for being lost to follow up due to gaps in transition and a paucity of adult treatment centers. As a result, many pediatric centers continue to follow 
Table 2 Recommendations for psychological testing

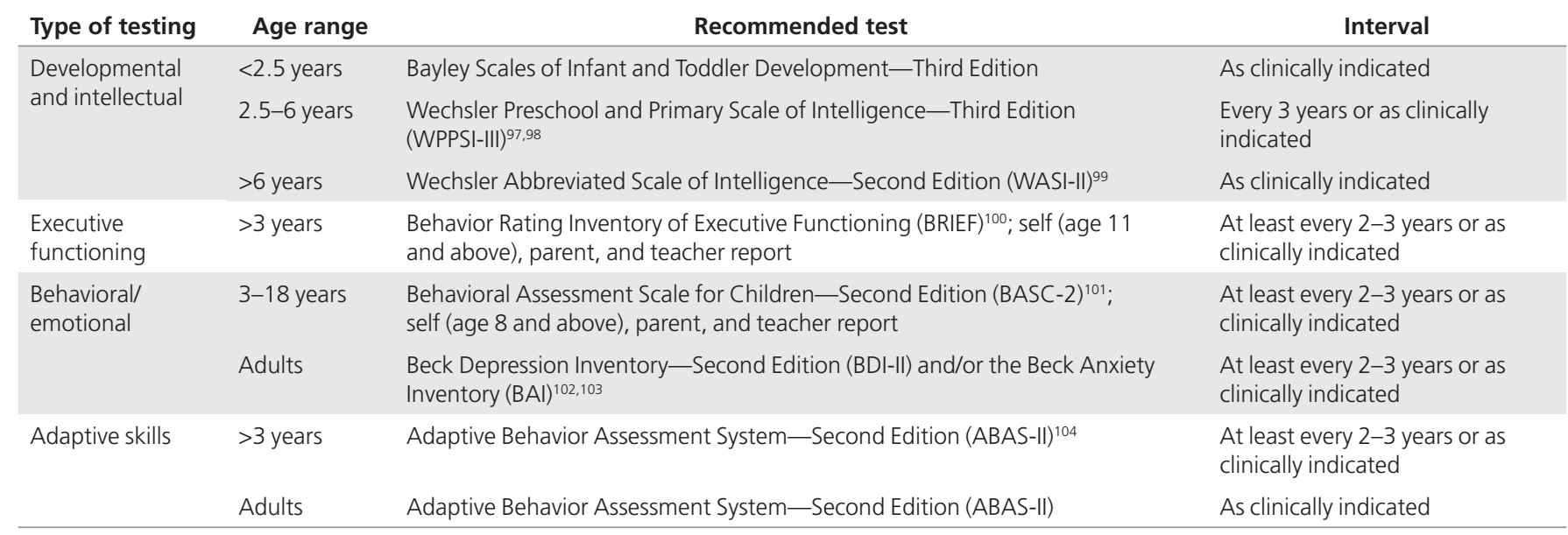

adult patients (often at pediatric hospitals), which does not foster independence. A further concern is raised by cost of therapy. Young adults are one of the most underinsured age groups in the United States. ${ }^{92}$ In addition, the cost of medical foods may not be covered by all insurance plans or other payers, adding a significant financial burden to patients who may already have difficulty with cognitive or executive functioning, impairing their ability to navigate the healthcare system. As treatment for life is recommended, third-party coverage of medical foods is critical.

Established protocols for transfer to adult centers in other diseases effectively reduce morbidity, and a tool kit for transition for individuals with PAH deficiency is available (http://newenglandconsortium.org/toolkit/). Transition should be a graduated process that begins in childhood and ends in early adulthood, allowing a gradual transfer of responsibility to patients and fostering the development of autonomy. ${ }^{93}$ Establishment of young adult or transitioning clinics that share adult and pediatric physicians may aid in this process. ${ }^{94,95}$ During transition, there should be emphasis on pregnancy-related issues for females. This should be discussed prior to adolescence and revisited annually. Without effective transitional care, many of the advantages gained through early treatment of PAH deficiency may be lost.

\section{KEY POINTS}

- Adult-focused treatment centers are optimal for older patients but are unavailable in many areas;

- Transition programs for adolescents with PAH deficiency should foster independence.

\section{RECOMMENDATIONS}

- Treatment for life mandates the need for medical insurance to provide coverage for medications and medical foods regardless of age.

\section{FUTURE DIRECTIONS}

As one of the longest-studied inborn errors of metabolism, $\mathrm{PAH}$ deficiency serves as a paradigm for the management of similar conditions, with enormous progress made in the understanding of this disorder and its treatment over the past decades. Asymptomatic newborns are now successfully diagnosed and treated, and treatment modalities are expanding to include pharmacological interventions. Moreover, large numbers of children have transitioned to adulthood, careers, and families. However, since the initial cohort of treated adults is only now reaching their 50s, there remains much to learn about PAH deficiency in older individuals.

$\mathrm{PAH}$ deficiency is now recognized as having a spectrum of severity with multiple contributing factors leading to ultimate outcome. This "paradigm shift" in our outlook on genetic diseases and their treatment began with PAH deficiency, but the journey is far from over. ${ }^{96}$ Looking forward, better tools and strategies are required to optimize care for the individual and improve long-term outcomes. Better biomarkers are also needed to monitor therapy and predict outcome. Current and future therapies should be evaluated not only for their ability to lower PHE but also for effects on enhancing quality of life for affected individuals and their families. Additional studies on treatment of individuals at the mildest end of the PAH-deficiency spectrum are necessary to define risks of blood PHE levels in the 360-600 $\mu \mathrm{mol} / \mathrm{l}$ range. It is critical for clinicians, scientists, insurers, and regulatory agencies to recognize the unique challenges in evaluating new therapies for rare disorders, such as PAH deficiency, and to develop novel methods to assess these interventions. An additional focus on understanding both genotype-phenotype relationships and modifiers of the PAH deficiency will allow individualization of therapy. New therapies, including gene therapy and hepatocyte transplant, have shown some efficacy in animal or limited human trials but require further development and validation for routine clinical use. Combination therapy including dietary and pharmacologic agents is likely to become the norm in PAH deficiency. Blood PHE is the current "gold standard" for monitoring treatment but is remote from the primary organ of interest (the brain). Real time, point-of-care PHE monitoring would provide more opportunity to engage patients in their own care. Effort should be made to better understand the mechanism of neurotoxicity of elevated PHE levels. Data on 
optimal control to prevent unwanted sequelae in adulthood are needed. The target PHE range of $<360 \mu \mathrm{mol} / 1$ in adolescence and adulthood needs further validation through continued collection of long-term clinical and patient-oriented outcome data.

\section{SUPPLEMENTARY MATERIAL}

Supplementary material is linked to the online version of the paper at http://www.nature.com/gim

\section{ACKNOWLEDGMENTS}

The Guideline Working Group gratefully acknowledges Dr. Paul Coates and Kathryn Camp of the Office of Dietary Supplements at the National Institutes of Health and the American College of Medical Genetics and Genomics with logistical support.

\section{DISCLOSURE}

J.V., H.C.A., J.M., and B.K.B. have participated in and received research funding for clinical trials related to $\mathrm{PAH}$ deficiency. H.C.A. serves on the advisory board for the PKUDOS sapropterin post-marketing patient registry. B.K.B. is a consultant for and serves on advisory boards for BioMarin Pharmaceuticals. K.M.A., N.E.B., D.M.F., W.E.S., B.H.T., and S.A.B. have no conflicts to declare relative to this guideline.

\section{REFERENCES}

1. Howell RR. National Institutes of Health Consensus Development Conference Statement: phenylketonuria: screening and management, October 16-18, 2000. Pediatrics 2001;108(4):972-982.

2. Lord J, Thomason MJ, Littlejohns P, et al. Secondary analysis of economic data: a review of cost-benefit studies of neonatal screening for phenylketonuria. J Epidemiol Community Health 1999;53:179-186.

3. Dhondt JL, Farriaux JP, Sailly JC, Lebrun T. Economic evaluation of cost-benefit ratio of neonatal screening procedure for phenylketonuria and hypothyroidism. $J$ Inherit Metab Dis 1991;14:633-639.

4. Lee PJ, Amos A, Robertson L, et al. Adults with late diagnosed phenylketonuria and severe challenging behavior: a randomized placebo-controlled trial of phenylalanine-restricted diet. J Neuro/ Neurosurg Psychiatry 2009;80(6):631635.

5. Mitchell JJ. Phenylalanine hydroxylase deficiency. In: Pagon RA, Adam MP, Bird TD, et al. (eds). GeneReviews ${ }^{T M}$ [Internet]. University of Washington, Seattle: Seattle, WA, 2000:1993-2013. http://www.ncbi.nlm.nih.gov/books/NBK1504/.

6. Kayaalp E, Treacy E, Waters PJ, Byck S, Nowacki P, Scriver CR. Human phenylalanine hydroxylase mutations and hyperphenylalaninemia phenotypes: a metanalysis of genotype-phenotype correlations. Am J Hum Genet 1997:61:1309-1317.

7. Scriver $\mathrm{CL}$, Kaufman S. Hyperphenylalaninemia: phenylalanine hydroxylase deficiency. In: Scriver C, Beaudet AL, Sly WS (eds). The Metabolic And Molecular Bases Of Inherited Disease. 8th ed. McGraw Hill: New York, NY, 2001:16671724.

8. Lindegren M, Krishnaswami S, Fonnesbeck C, et al. Adjuvant Treatment for Phenylketonuria (PKU). Comparative Effectiveness Review No. 56. AHRQ Publication: Rockville, MD, 2012:1-343.

9. Vockley J, Chapman KA, Arnold GL. Development of clinical guidelines for inborn errors of metabolism: commentary. Mol Genet Metab 2013;108:203205.

10. Guthrie R, Susi A. A simple phenylalanine method for detecting phenylketonuria in large populations of newborn infants. Pediatrics 1963;32:338-343.

11. Chace DH, Millington DS, Terada N, Kahler SG, Roe CR, Hofman LF. Rapid diagnosis of phenylketonuria by quantitative analysis for phenylalanine and tyrosine in neonatal blood spots by tandem mass spectrometry. Clin Chem 1993;39:66-71

12. Shapira EBM, Miller J, Africk D. Plasma Amino Acid Analysis. Biochemical Genetics: A Laboratory Manual. Oxford Press: New York, NY; 1989.
13. McHugh D. Clinical validation of cutoff target ranges in newborn screening of metabolic disorders by tandem mass spectrometry: a worldwide collaborative project. Genet Med 2011;13:230-254.

14. Marquardt $G$, Rinaldo $P$. Enhanced interpretation of newborn screening results without analyte cutoff values. Genet Med 2012;14:648-655.

15. Norman R, Haas M, Wilcken B. International perspectives on the costeffectiveness of tandem mass spectrometry for rare metabolic conditions. Health Policy 2009;89:252-260.

16. Blau N, Hennermann JB, Langenbeck U, Lichter-Konecki U. Diagnosis, classification, and genetics of phenylketonuria and tetrahydrobiopterin (BH4) deficiencies. Mol Genet Metab 2011;104:S2-S9.

17. Blau N, Burgard P. Disorders of Phenylalanine and Tetrahydrobiopterin. Physicians' Guide to the Treatment and Follow-up of Metabolic Diseases. Springer: Heidelberg; 2005:89-105.

18. Scriver CR, Waters PJ. Monogenic traits are not simple: lessons from phenylketonuria. Trends Genet 1999;15:267-272.

19. Levy HL, Milanowski A, Chakrapani A, et al.; Sapropterin Research Group. Efficacy of sapropterin dihydrochloride (tetrahydrobiopterin, 6R-BH4) for reduction of phenylalanine concentration in patients with phenylketonuria: a phase III randomised placebo-controlled study. Lancet 2007;370: 504-510.

20. Güttler F, Guldberg P. Mutation analysis anticipates dietary requirements in phenylketonuria. Eur J Pediatr 2000;159(suppl 2):S150-S153.

21. Zschocke J, Hoffmann GF. PAH gene mutation analysis in clinical practicecomments on mutation analysis anticipates dietary requirements in phenylketonuria. Eur J Pediatr 2000;159(suppl 2):S154-S155.

22. Greeves LG, Patterson CC, Carson DJ, et al. Effect of genotype on changes in intelligence quotient after dietary relaxation in phenylketonuria and hyperphenylalaninaemia. Arch Dis Child 2000;82:216-221.

23. Trefz FK, Scheible D, Götz H, Frauendienst-Egger G. Significance of genotype in tetrahydrobiopterin-responsive phenylketonuria. J Inherit Metab Dis 2009;32:22-26

24. Weglage J, Ullrich $K$, Pietsch $M$, Fünders B, Zass R, Koch HG. No fine motor deficits in patients with untreated non-phenylketonuria hyperphenylalaninaemia. Acta Paediatr 1996;85:320-323.

25. Costello PM, Beasley MG, Tillotson SL, Smith I. Intelligence in mild atypical phenylketonuria. Eur J Pediatr 1994;153:260-263.

26. Gassió R, Artuch R, Vilaseca MA, et al. Cognitive functions in classic phenylketonuria and mild hyperphenylalaninaemia: experience in a paediatric population. Dev Med Child Neurol 2005;47:443-448.

27. Diamond A, Prevor M, Callender G, Druin DP. Prefrontal cortex cognitive deficits in children treated early and continuously for PKU. Monographs Soc Res Child Dev 1977:62(4):1-206.

28. Weglage J, Pietsch $M$, Feldmann $R$, et al. Normal clinical outcome in untreated subjects with mild hyperphenylalaninemia. Pediatr Res 2001;49:532-536.

29. Singh RH, Rohr F, Frazier $D$, et al. Recommendations for the nutrition management of phenylalanine hydroxylase deficiency. Genet Med, e-pub ahead of print 2 January 2014

30. Ney DM, Gleason ST, vanCalcar SC, et al. Nutrition management of PKU with glycomacropeptide from cheese whey. J Inherit Metab Dis 2009;321:32-39.

31. Singh R, Acosta P, Burton B, et al. Tracking Long-term Outcomes: Development of Care Data Elements (CDE) for Phenylketonuria. 4th GMDI Edication Conference: New Orleans, LA, 2012.

32. Gregory CO, Yu C, Singh RH. Blood phenylalanine monitoring for dietary compliance among patients with phenylketonuria: comparison of methods. Genet Med 2007;9:761-765.

33. Modan-Moses D, Vered I, Schwartz G, et al. Peak bone mass in patients with phenylketonuria. J Inherit Metab Dis 2007;30:202-208.

34. Burton BK, Grange DK, Milanowski A, et al. The response of patients with phenylketonuria and elevated serum phenylalanine to treatment with oral sapropterin dihydrochloride (6R-tetrahydrobiopterin): a phase II, multicentre, open-label, screening study. J Inherit Metab Dis 2007;30: 700-707.

35. Ziesch B, Weigel J, Thiele A, et al. Tetrahydrobiopterin $(B H(4))$ in PKU: effect on dietary treatment, metabolic control, and quality of life. J Inherit Metab Dis 2012;35(6):983-992.

36. Leuret $\mathrm{O}$, Barth $\mathrm{M}$, Kuster $\mathrm{A}$, et al. Efficacy and safety of $\mathrm{BH} 4$ before the age of 4 years in patients with mild phenylketonuria. J Inherit Metab Dis 2012;35:975981.

37. Utz JR, Lorentz CP, Markowitz D, et al. START, a double blind, placebo-controlled pharmacogenetic test of responsiveness to sapropterin dihydrochloride in phenylketonuria patients. Mol Genet Metab 2012;105:193-197. 
38. Gordon P, Thomas JA, Suter R, Jurecki E. Evolving patient selection and clinical benefit criteria for sapropterin dihydrochloride (Kuvan $\AA$ ) treatment of PKU patients. Mol Genet Metab 2012;105:672-676.

39. Cunningham A, Bausell H, Brown M, et al. Recommendations for the use of sapropterin in phenylketonuria. Mol Genet Metab 2012;106:269-276.

40. Shintaku H, Ohwada M. Long-term follow-up of tetrahydrobiopterin therapy in patients with tetrahydrobiopterin deficiency in Japan. Brain Dev 2012;35(5):406-410.

41. Trefz FK, Burton BK, Longo N, et al.; Sapropterin Study Group. Efficacy of sapropterin dihydrochloride in increasing phenylalanine tolerance in children with phenylketonuria: a phase III, randomized, double-blind, placebo-controlled study. J Pediatr 2009;154:700-707.

42. Matalon R, Michals-Matalon K, Bhatia G, et al. Large neutral amino acids in the treatment of phenylketonuria (PKU). J Inherit Metab Dis 2006;29:732738.

43. Longo N, Burton B, Harding C, et al. A Phase 2, Open-Label, Dose-Finding and the Long Term Extension Study to Evaluate the Safety, Efficacy and Tolerability of Multiple Subcutaneous Doses of rAvPAL-PEG in Patients With Phenylketonuria. American College of Medical Genetics and Genomics 2012 Annual Meeting: Charlotte, NC, 2012.

44. Brumm VL, Bilder D, Waisbren SE. Psychiatric symptoms and disorders in phenylketonuria. Mol Genet Metab 2010;99(suppl 1):S59-S63.

45. Smith I, Beasley MG, Wolff OH, Ades AE. Behavior disturbance in 8-year-old children with early treated phenylketonuria. Report from the MRC/DHSS Phenylketonuria Register. J Pediatr 1988;112:403-408.

46. Smith I, Knowles J. Behaviour in early treated phenylketonuria: a systematic review. Eur J Pediatr 2000;159(suppl 2):S89-S93.

47. Sullivan JE, Chang P. Review: emotional and behavioral functioning in phenylketonuria. J Pediatr Psychol 1999;24:281-299.

48. Waisbren SE, Levy HL. Agoraphobia in phenylketonuria. J Inherit Metab Dis 1991;14:755-764

49. Burton BK, Leviton L, Vespa $\mathrm{H}$, et al. A diversified approach for PKU treatment: routine screening yields high incidence of psychiatric distress in phenylketonuria clinics. Mol Genet Metab 2013;108:8-12.

50. Simon E, Schwarz M, Roos J, et al. Evaluation of quality of life and description of the sociodemographic state in adolescent and young adult patients with phenylketonuria (PKU). Health Qual Life Outcomes 2008;6:25.

51. Bosch AM, Tybout W, van Spronsen FJ, de Valk HW, Wijburg FA, Grootenhuis MA. The course of life and quality of life of early and continuously treated Dutch patients with phenylketonuria. J Inherit Metab Dis 2007;30:29-34.

52. Demirkol M, Gizewska M, Giovannini M, Walter J. Follow up of phenylketonuria patients. Mol Genet Metab 2011;104:S31-S39.

53. Rohr FJ, Doherty LB, Waisbren SE, et al. New England Maternal PKU Project: prospective study of untreated and treated pregnancies and their outcomes. $J$ Pediatr 1987;110:391-398.

54. Brown AS, Fernhoff PM, Waisbren SE, et al. Barriers to successful dietary control among pregnant women with phenylketonuria. Genet Med 2002;4:84-89.

55. Ng TW, Rae A, Wright H, Gurry D, Wray J. Maternal phenylketonuria in Western Australia: pregnancy outcomes and developmental outcomes in offspring. J Paediatr Child Health 2003;39:358-363.

56. Matalon KM, Acosta PB, Azen C. Role of nutrition in pregnancy with phenylketonuria and birth defects. Pediatrics 2003;112(6 Pt 2):1534-1536.

57. Tessier R, Nowak E, Assoun M, et al. Maternal phenylketonuria: low phenylalanine might increase the risk of intra uterine growth retardation. J Inherit Metab Dis 2012; 35:993-999.

58. Lee PJ, Ridout D, Walter JH, Cockburn F. Maternal phenylketonuria: report from the United Kingdom Registry 1978-97. Arch Dis Child 2005;90:143-146.

59. Koch R, Hanley W, Levy H, et al. Maternal phenylketonuria: an international study. Mol Genet Metab 2000;71:233-239.

60. Bradburn NC, Wappner RS, Lemons JA, Meyer BA, Roberts RS. Lactation and phenylketonuria. Am J Perinatol 1985:2:138-141.

61. Fox-Bacon C, McCamman S, Therou L, Moore W, Kipp DE. Maternal PKU and breastfeeding: case report of identical twin mothers. Clin Pediatr (Phila) 1997;36:539-542.

62. Srinivasan BS, Evans EA, Flannick J, et al. A universal carrier test for the long tail of Mendelian disease. Reprod Biomed Online 2010;21:537-551.

63. Mallolas J, Milà M, Lambruschini N, Cambra FJ, Campistol J, Vilaseca MA. Biochemical phenotype and its relationship with genotype in hyperphenylalaninemia heterozygotes. Mol Genet Metab 1999;67:156-161.

64. Pietz J, Dunckelmann R, Rupp A, et al. Neurological outcome in adult patients with early-treated phenylketonuria. Eur J Pediatr 1998;157:824-830.
65. Burgard P. Development of intelligence in early treated phenylketonuria. Eur J Pediatr 2000;159(suppl 2):S74-S79.

66. Koch R, Burton B, Hoganson G, et al. Phenylketonuria in adulthood: a collaborative study. J Inherit Metab Dis 2002;25:333-346.

67. Waisbren SE, Schnell RR, Levy HL. Diet termination in children with phenylketonuria: a review of psychological assessments used to determine outcome. J Inherit Metab Dis 1980;3:149-153.

68. Azen C, Koch R, Friedman E, Wenz E, Fishler K. Summary of findings from the United States Collaborative Study of children treated for phenylketonuria. Eur J Pediatr 1996;155 Suppl 1:S29-S32.

69. Fishler K, Azen CG, Henderson R, Friedman EG, Koch R. Psychoeducational findings among children treated for phenylketonuria. Am J Ment Defic 1987:92:65-73

70. Moyle JJ, Fox AM, Arthur M, Bynevelt M, Burnett JR. Meta-analysis of neuropsychological symptoms of adolescents and adults with PKU. Neuropsychol Rev 2007;17:91-101.

71. Sternberg RJ, Grigorenko EL. Intelligence and culture: how culture shapes what intelligence means, and the implications for a science of well-being. Philos Trans $R$ Soc Lond, B, Biol Sci 2004;359:1427-1434

72. Pennington BF, van Doorninck WJ, McCabe LL, McCabe ER. Neuropsychological deficits in early treated phenylketonuric children. Am J Ment Defic 1985;89:467-474

73. Welsh MC, Pennington BF, Ozonoff S, Rouse B, McCabe ER. Neuropsychology of early-treated phenylketonuria: specific executive function deficits. Child Dev 1990;61:1697-1713.

74. Weglage J, Ullrich K, Pietsch M, Fünders B, Zass R, Koch HG. Untreated nonphenylketonuric-hyperphenylalaninaemia: intellectual and neurological outcome. Eur J Pediatr 1996;155 Suppl 1:S26-S28.

75. Huijbregts SC, de Sonneville LM, van Spronsen FJ, Licht R, Sergeant JA. The neuropsychological profile of early and continuously treated phenylketonuria: orienting, vigilance, and maintenance versus manipulation-functions of working memory. Neurosci Biobehav Rev 2002;26:697-712.

76. Stemerdink BA, van der Meere JJ, van der Molen MW, et al. Information processing in patients with early and continuously-treated phenylketonuria. Eur J Pediatr 1995;154:739-746.

77. Ris MD, Williams SE, Hunt MM, Berry HK, Leslie N. Early-treated phenylketonuria: adult neuropsychologic outcome. J Pediatr 1994;124:388392

78. Christ SE, Huijbregts SC, de Sonneville LM, White DA. Executive function in early-treated phenylketonuria: profile and underlying mechanisms. Mol Genet Metab 2010;99(suppl 1):S22-S32.

79. Sharman R, Sullivan K, Young R, McGill J. Biochemical markers associated with executive function in adolescents with early and continuously treated phenylketonuria. Clin Genet 2009;75:169-174

80. Brumm VL, Grant ML. The role of intelligence in phenylketonuria: a review of research and management. Mol Genet Metab 2010;99 (suppl 1):S18-S21.

81. Gassió R, Fusté E, López-Sala A, Artuch R, Vilaseca MA, Campistol J. School performance in early and continuously treated phenylketonuria. Pediatr Neurol 2005;33:267-271

82. Antshel KM, Waisbren SE. Timing is everything: executive functions in children exposed to elevated levels of phenylalanine. Neuropsychology 2003;17:458468.

83. Channon S, German E, Cassina C, Lee P. Executive functioning, memory, and learning in phenylketonuria. Neuropsychology 2004;18:613-620.

84. Smith I, Beasley MG, Ades AE. Intelligence and quality of dietary treatment in phenylketonuria. Arch Dis Child 1990;65:472-478.

85. DeRoche K, Welsh M. Twenty-five years of research on neurocognitive outcomes in early-treated phenylketonuria: intelligence and executive function. Dev Neuropsychol 2008;33:474-504

86. Waisbren SE, Noel K, Fahrbach K, et al. Phenylalanine blood levels and clinical outcomes in phenylketonuria: a systematic literature review and meta-analysis. Mol Genet Metab 2007:92:63-70.

87. Antshel KM, Waisbren SE. Developmental timing of exposure to elevated levels of phenylalanine is associated with ADHD symptom expression. J Abnorm Child Psychol 2003;31:565-574.

88. Arnold GL, Vladutiu CJ, Orlowski CC, Blakely EM, DeLuca J. Prevalence of stimulant use for attentional dysfunction in children with phenylketonuria. $J$ Inherit Metab Dis 2004:27:137-143.

89. Gentile JK, Ten Hoedt AE, Bosch AM. Psychosocial aspects of PKU: hidden disabilities-a review. Mol Genet Metab 2010;99(suppl 1):S64-S67. 
90. Kazak AE, Reber M, Snitzer L. Childhood chronic disease and family functioning a study of phenylketonuria. Pediatrics 1988;81:224-230.

91. Waisbren S, White DA. Screening for cognitive and social-emotional problems in individuals with PKU: tools for use in the metabolic clinic. Mol Genet Metab 2010;99(suppl 1):S96-S99.

92. DeNavas-Walt C, Proctor BD, Smith JC. U.S. Census Bureau, Current Population Reports, P60-239. Income, Poverty, and Health Insurance Coverage in the United States: 2010. U.S. Government Printing Office: Washington, DC, 2011.

93. American Academy of Pediatrics, American Academy of Family Physicians, American College of Physicians, Transitions Clinical Report Authoring Group, Cooley WC, Sagerman PJ. Supporting the health care transition from adolescence to adulthood in the medical home. Pediatrics 2011;128:182-200.

94. Crowley R, Wolfe I, Lock K, McKee M. Improving the transition between paediatric and adult healthcare: a systematic review. Arch Dis Child 2011;96:548-553.

95. Harden PN, Walsh G, Bandler N, et al. Bridging the gap: an integrated paediatric to adult clinical service for young adults with kidney failure. BMJ 2012;344:e3718.
96. Scriver CR. The PAH gene, phenylketonuria, and a paradigm shift. Hum Mutat 2007;28:831-845.

97. Bayley N. Bayley Scales of Infant and Toddler Development - Third Edition (Bayley-III). Pearson Publishing: San Antonio, TX; 2005.

98. Wechsler D. Wechsler Preschool and Primary Scale of Intelligence - Third Edition (WPPSI-III). Pearson Publishing: San Antonio, TX; 2002.

99. Wechsler D. Wechsler Abbreviated Scale of Intelligence - 2nd edition (WASI-II). Pearson Publishing: San Antonio, TX; 2011.

100. Gioia GA, Peter K, Guy S, Kenworthy L. Behavior Rating Inventory of Executive Functioning (BRIEF). PAR: Lutz, FL; 2000.

101. Kamphaus RW. The Behavioral Assessment System for Children - Second edition. American Guidance Service: Circle Pines, MN; 2005.

102. Beck AT, Steer RA, Brown G. Beck Depression Inventory - 2nd edition (BDI-II). Pearson Publishing: San Antonio, TX; 1996.

103. Beck A. Beck Anxiety Inventory (BAI). Pearson Publishing: San Antonio, TX 1993.

104. Harrison P, Oakland T. Adaptive Behavior Assessment System-Second Edition (ABAS-II). Pearson Publishing: San Antonio, TX; 2003. 